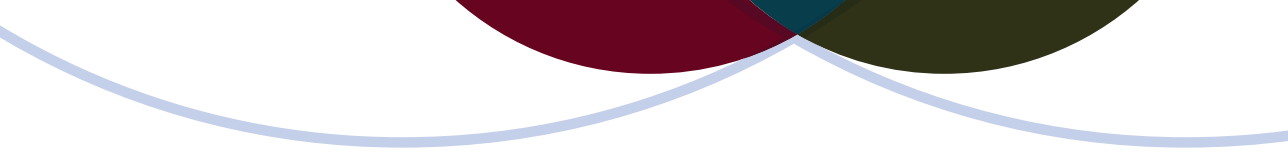

\author{
ARTÍCULO DE INVESTIGACIÓN \\ RESEARCH REPORT
}

\title{
Cuando leer y escribir conspiran con los sentidos y las palabras se sumergen en la música el texto escrito cobra vida
}

\author{
Claudia marcela callejas Velasco \\ Universidad Autónoma de Bucaramanga, Colombia \\ Orcid: https://orcid.org/0000-0002-8487-6442 \\ Autor de correspondencia: https://orcid.org/0000-0002-0101-9489 \\ Claudia Lucia Rugeles Rey \\ Universidad Autónoma de Bucaramanga, Colombia \\ Orcid: https://orcid.org/0000-0002-0101-9489
}


Desde la etapa inicial del desarrollo humano, la preocupación primordial en los primeros años de vida escolar es el proceso de lectura y escritura. Si bien es cierto que leer y escribir son dos habilidades que van de la mano en el proceso de aprendizaje de los escolares, es indispensable centrar especial interés en ejercicios pedagógicos que enriquezcan el desempeño de estos procesos comunicativos en diferentes contextos sociales.

Es fundamental que, para lograr mejores resultados en el proceso de lectura y escritura escolar, el docente deba impactar el sistema nervioso de los estudiantes, activando la inteligencia, que es la clave que permite solucionar problemas mediante el aprendizaje. Así es como se conectan los sentidos con la realidad, para promover el conocimiento.

De esta manera, se establece la pertinencia de la música como medio para captar la atención, las emociones, la deducción y la reflexión del mensaje que traen las letras de las canciones, a fin de generar nuevos textos que plasmen sus propias ideas, emociones y experiencias de vida. Por ello, la música se convierte en un vínculo entre la realidad y los intereses de los estudiantes, porque puede ser usada como material didáctico para trabajar aspectos léxicos, gramaticales, fonéticos y culturales.

Palabras clave: producción escrita; inteligencia emocional; canciones populares; texto narrativo; historias de vida.

\section{A B S T R A C T}

The initial stage of human development, the main concern in the early years of school life is the process of reading and writing. While it is true that reading and writing are two skills that go hand in hand in the student's learning process, it is essential to focus on the special pedagogical interest exercises can enhance the performance of the communication process in different social contexts.

It is fundamental to achieve better results in the process of reading and writing school skills, the teacher must impact the nervous system of students by activating intelligence, which is the key to solve problems through the learning process. This is how the senses can be connected with reality, to promote knowledge.

In this way, the pertinence of music is established to capture the attention, the emotions, the deduction and the reflection of the message that bring the lyrics of the songs to generate new texts that capture their own ideas, the emotions and the lifetime experiences. Because of them, music becomes a link between reality and the interests of students and it can be used with didactic material to work on lexical, grammatical, phonetic and cultural aspects.

Key words: Written production, emotional intelligence, popular songs, narrative text, life stories.

Como citar este artículo:

Callejas Velasco, C. \& Rugeles Rey, C. (2020). Cuando leer y escribir conspiran con los sentidos y las palabras se sumergen en la música el texto escrito cobra vida. Zona Proxima, 33, 125-145. 
Cuando leer y escribir conspiran con los sentidos y las palabras

se sumergen en la música el texto escrito cobra vida

Claudia Marcela Callejas Velasco, Claudia Lucia Rugeles Rey

\section{INTRODUCCIÓN}

Leer y escribir son habilidades comunicativas primordiales en el ser humano, en cada una de ellas se evidencian procesos de pensamiento que implican el ejercicio competente del uso del lenguaje en diferentes contextos.

Enseñar a leer siempre ha sido una de las preocupaciones educativas constantes y por ello ha implicado cambios en los procesos de enseñanza. Ahora bien, leer de manera comprensiva es también causa de infinidad de trabajos investigativos, porque generalmente la comprensión de lectura llega al nivel literal, lo cual evidencia con mayor dificultad el nivel inferencial y el críticointertextual.

Escribir es más que la codificación de significados en atención a reglas lingüísticas, es un proceso individual y social en el que se evidencia la competencia comunicativa en su sentido más amplio y requiere procesos de pensamiento más complejos, como decodificar, memorizar e inferir, que se expresan en la intención comunicativa. Constantemente, ha sido un proceso alejado del contexto de los estudiantes, exclusivo de la enseñanza de reglas ortográficas, y estudiado como un producto y no como un proceso de interacción y coevaluación.

Si bien es cierto que leer y escribir son dos habilidades que van de la mano en el proceso de aprendizaje de los escolares, es indispensable centrar especial interés en ejercicios pedagógicos que enriquezcan el desempeño de estos procesos comunicativos, en diferentes contextos sociales.

En el área de lengua castellana, se observan falencias en el proceso de aprendizaje, que impiden el desarrollo de las competencias comunicativas en los estudiantes. Leer, hablar, escuchar y escribir son ejercicios de la vida diaria que requieren especial atención en la producción textual, ya que en la institución educativa (IE) no se hacen visibles estrategias que ayuden a desarrollarlas eficazmente.

Orientados en el desarrollo de habilidades comunicativas, se lleva a cabo una propuesta de intervención pedagógica enfocada en fortalecer el proceso lectoescritor, motivada en la comprensión del mensaje de canciones populares, que sensibilizan las emociones de los estudiantes, las relacionan con un texto narrativo, y así evidencian la creación de escritos que surjan de sus experiencias de vida.

De esta manera, toda enseñanza que pretenda un verdadero aprendizaje debe partir del contexto, de las necesidades y de los intereses de los estudiantes, especialmente en ambientes escolares fuera de la rutina. 
Cuando leer y escribir conspiran con los sentidos y las palabras

se sumergen en la música el texto escrito cobra vida

Claudia Marcela Callejas Velasco, Claudia Lucia Rugeles Rey

\section{REFLEXIÓN EN TORNO AL MARCO TEÓRICO}

La ejecución del trabajo se lleva a cabo mediante la investigación-acción cualitativa. Para el desarrollo de la propuesta de investigación, fueron relevantes los aportes teóricos en tres líneas: conceptual, psicológico y crítico, que se relacionan en diferentes aspectos. En lo conceptual, las teorías de Cassany (2017), quien ve al lector como "producto social", plantea la escritura desde el enfoque comunicativo, que prioriza el aprendizaje de uso verbal en contextos significativos para el estudiante.

En la práctica educativa planeada para la enseñanza de las habilidades comunicativas, las actividades escolares que involucran a los estudiantes en el desarrollo de procesos que orientan la comprensión y producción textual están generalmente aisladas de su realidad, lo que genera apatía frente a estas. En este sentido, es pertinente citar a Cassany (2014), quien define la escritura como un proceso de composición acompañado de la contextualización de lo que se vive, en situaciones comunicativas reales. Es decir, el proceso de lectura y escritura es un proceso dinámico y abierto, puesto que el mensaje se construye a partir de la interacción entre los presaberes y los signos escritos, y de esta manera es el lector quien da sentido al texto, de acuerdo con sus experiencias; la voz del escritor no será la única voz referenciada. Cassany (2014) también refiere la importancia del educador, quien debe formar ciudadanos críticos, que opten por una posición crítica frente a lo que aprendan. Para ello, son necesarias las habilidades lingüísticas del lector.

Además, la propuesta se apoya en los aportes conceptuales sobre las estrategias de lectura de Solé (1998), "leer es un proceso de interacción entre el lector y el texto, siempre debe existir un objetivo que guíe la lectura” (p. 17).

Hacer lectores autónomos significa también hacer lectores capaces de aprender a partir de los textos. Para ello, quien lee debe ser capaz de interrogarse acerca de su propia comprensión, establecer relaciones entre lo que lee y lo que forma parte de su acervo personal, cuestionar su conocimiento y modificarlo, establecer generalizaciones que permitan transferir lo aprendido a otros contextos distintos (Solé, 1998). Por tanto, si un estudiante comprende de manera autónoma lo que lee, se evidencia este aprendizaje en la producción textual aplicado a diferentes contextos.

En la línea psicológica, los postulados de Goleman (2007) son pertinentes, ya que, para motivar el proceso de escritura, es necesario que las composiciones nazcan desde los intereses de los estudiantes y generalmente desde las propias experiencias de vida, que tocan los sentimientos y las emociones, a veces difíciles de expresar. Goleman explica que dentro de su enfoque sobre la inteligencia emocional existen dimensiones básicas que la vertebran: la autoconciencia, la capacidad 
Cuando leer y escribir conspiran con los sentidos y las palabras

se sumergen en la música el texto escrito cobra vida

Claudia Marcela Callejas Velasco, Claudia Lucia Rugeles Rey

para entender lo que sentimos y estar conectados con los valores, a nuestra esencia; la automotivación, la habilidad para orientarnos hacia nuestras metas, de recuperarse de los contratiempos; y la conciencia social y la empatía, la habilidad para relacionarnos, para comunicar, para llegar a acuerdos, para conectar positiva y respetuosamente con los demás (p. 64).

Dentro de los aportes en la línea crítica, según Van Dijk, citado por Cassany (2017), una teoría empírica de la lengua también necesita modelos que expliquen cómo se comprende y cómo se usa el discurso en la comunicación. Van Dijk deja claro que, en el análisis del discurso, el contexto desempeña un papel fundamental en la descripción y explicación de los textos escritos y orales, en el cual se distinguen estructuras como los ambientes, los participantes y sus roles sociocomunicativos, las intenciones, las metas o los propósitos.

Es fundamental que, para lograr mejores resultados en el proceso de lectura y escritura escolar, el docente impacte el sistema nervioso de los estudiantes mediante la comprensión y reflexión de las historias inmersas en las canciones populares con las cuales ellos se identifican, activen la inteligencia, que es la clave que permite solucionar problemas mediante el aprendizaje y conecten los sentidos con la realidad para promover el conocimiento.

Es de esta manera que la música se emplea como un recurso atractivo, favorece el aprendizaje debido a la importancia que tiene en la vida, permite un acercamiento entre docente y estudiantes, y genera una relación de interacción y cooperación.

\section{LA MÚSICA COMO HERRAMIENTA PEDAGÓGICA}

Para que leer se torne placentero y escribir se convierta en una herramienta de creación de mundos posibles, es necesario involucrar los intereses de los estudiantes. De ahí que se piensen y planifiquen herramientas pedagógicas que motiven y propicien las prácticas hacia el mejoramiento de estos procesos.

De acuerdo con Díaz, Morales y Díaz (2014), la música, además de ser una expresión artística, puede ser utilizada como recurso pedagógico que favorezca el desarrollo intelectual, motor y de lenguaje en los niños, a través del fortalecimiento de procesos cognitivos, como la memoria, la atención, la percepción y la motivación.

En este sentido, se establece la pertinencia de la música como medio para atrapar la atención, las emociones, la deducción y la reflexión del mensaje que traen las letras de las canciones, para 
Cuando leer y escribir conspiran con los sentidos y las palabras

se sumergen en la música el texto escrito cobra vida

Claudia Marcela Callejas Velasco, Claudia Lucia Rugeles Rey

generar nuevos textos que plasmen sus propias ideas, emociones y experiencias de vida. Por ello, la música se convierte en un vínculo entre la realidad y los intereses de los estudiantes.

Involucrar la música en el aprendizaje aumenta el interés, permite la expresión de sentimientos y la cooperación. La música hace posible que un grupo se reúna alrededor de una canción porque conecta las emociones y trae a la mente recuerdos que en ocasiones no son expresados con facilidad.

El método Dalcroze (Bachmann, 1988) es un referente teórico que tiende a una educación integral, porque trabaja simultáneamente la atención, la inteligencia, la rapidez mental, la sensibilidad y el movimiento. A través de la música se despiertan las emociones, lo que permite atraer la atención, activar la inteligencia y expresar sensibilidad. El ritmo, el movimiento y la danza son elementos principales del método Dalcroze, considera la unión de mente y cuerpo, de modo que es el cuerpo un mediador entre el sonido, el pensamiento y el sentimiento. "La música es oída por nuestros oídos, pero escuchada con todo el cuerpo".

La música no debe estar limitada solo al estudio musical. Todas las personas escuchan música, tienen un artista, género o canción con la que se identifican. Permitir que la música entre en las clases genera acercamiento con los estudiantes, favorece la interacción y la cooperación. Mediante el disfrute de una canción, conocemos sentimientos, reflexionamos sobre el mensaje, trabajamos vocabulario y favorecemos la confianza, la participación y trabajo en grupo, el respeto por las ideas y los sentimientos de los demás.

El aprendizaje de la música se relaciona con la escritura en varios aspectos. "La lectura de una partitura, por ejemplo, utiliza el mismo proceso cerebral del lado izquierdo del cerebro que la lectura de un texto", dice el profesor Fernando Capovilla, citado por Bertolini. "Además, se activa la región prefrontal del cerebro, relacionado con los conceptos de tiempo y motricidad, esta región es la que posee el mayor número de neuronas en el cerebro" (Bertolini, 2015).

En un estudio realizado sobre cómo el cerebro aprende a leer y escribir, la maestra Fräulein Paula del Departamento de Psicología del Aprendizaje, Desarrollo y Personalidad de la Universidad de São Paulo, explica que el lenguaje es una antigua habilidad en nuestra filogenia, mientras que la escritura es una nueva forma de conocimiento. La música, además de contribuir a un aprendizaje estético, es un regulador emocional, que tiene grandes efectos sobre el desarrollo de ciertos mecanismos del pensamiento.

Con los referentes mencionados, en el desarrollo de la propuesta de intervención pedagógica, resulta más atractivo para los estudiantes escribir textos narrativos a partir de sus propias expe- 
Cuando leer y escribir conspiran con los sentidos y las palabras

se sumergen en la música el texto escrito cobra vida

Claudia Marcela Callejas Velasco, Claudia Lucia Rugeles Rey

riencias. Empleando la música como instrumento de motivación para incentivar la lectura y la escritura, se pretende que los estudiantes lean y analicen las letras de las canciones que escuchan, generen un espacio de reflexión y pongan en juego habilidades como hablar y escuchar para llegar al momento final de escritura y creación, en que los estudiantes sean quienes produzcan nuevas historias movidos por sus emociones y pensamientos.

\section{METOdOlOGÍA}

La propuesta tuvo lugar en el municipio de Piedecuesta, en el departamento de Santander, en una IE de carácter oficial que ofrece los niveles de preescolar, básica y media, a una población aproximada de 3000 estudiantes cuyas edades oscilan entre 5 y 18 años, pertenecientes en su mayoría a familias de nivel socioeconómico de estratos 1, 2 y 3. En la jornada de la mañana, desde las 5:50 a. m. hasta las 12:10 p. m., se atiende a los estudiantes de básica secundaria, y en la jornada de la tarde desde las 12:15 p. m. hasta las 5:40 p. m. a los estudiantes de preescolar y básica primaria. Para ejecutar la propuesta de intervención pedagógica, se seleccionó un curso de 31 estudiantes de los cuatro cursos que conforman el grado décimo, y un curso de 31 estudiantes de los tres que conforman el grado undécimo.

La propuesta se desarrolló en los periodos de clase, enfocada en fortalecer el proceso lectoescritor, motivada en la comprensión y en el análisis de canciones populares; proceso que permite a los estudiantes elaborar juicios críticos y crear nuevos textos de acuerdo con el mensaje abordado en cada canción. Para ello, se implementó la planeación de cada clase mediante secuencias didácticas diseñadas según los estándares y derechos básicos de aprendizaje (DBA) en el área de lengua castellana, y estructuradas a la luz de los referentes teóricos expuestos por Daniel Cassany y Daniel Goleman, cada una con el objetivo de buscar la producción escrita en los estudiantes, siguiendo un proceso que parte de la motivación orientada hacia la planificación de la escritura.

Las sesiones de aprendizaje se diseñaron mediante secuencias didácticas estructuradas en seis momentos:

- Activar los sentidos

- Leer como un escritor

- Enfoque comunicativo

- La composición a partir de la interacción y el diálogo 
- Escritura

- Lectura grupal y coevaluación

Las secuencias constan de diferentes actividades establecidas de la siguiente manera: un primer momento de activación de los sentidos, en el que se presentan preguntas orientadas a la reflexión y el análisis alrededor de la canción escuchada; un segundo momento llamado "leer como un escritor", en que se propone un texto narrativo que mantenga alguna relación con la canción, para hacer un análisis de acuerdo con los tres niveles de comprensión textual (literal, inferencial, crítico); el tercer momento es el enfoque comunicativo orientado hacia una reflexión lingüística en la que se identifican saberes cotidianos en atención a los temas abordados en el texto y la canción; para el cuarto momento, la composición a partir de la interacción y el diálogo, en que se planifica la escritura con una lluvia de ideas y selección de frases paradigmáticas que surgen de las actividades anteriores; en el quinto momento de escritura, se inicia la creación de textos narrativos con la elaboración de borradores; y, finalmente, la lectura individual y grupal para revisar y hacer las correcciones necesarias que arrojan los resultados de la evaluación del texto por medio de la rúbrica, lo cual permite una coevaluación asertiva de todo el proceso de composición escrita, que concluye en un texto narrativo bien elaborado (tabla 1).

Tabla 1. Secuencia didáctica 3. 
Cuando leer y escribir conspiran con los sentidos y las palabras

se sumergen en la música el texto escrito cobra vida

Claudia Marcela Callejas Velasco, Claudia Lucia Rugeles Rey

\begin{tabular}{|c|c|c|}
\hline & & \\
\hline \multicolumn{3}{|c|}{ DESARROLLO DE ACTIVIDADES } \\
\hline \multicolumn{3}{|c|}{$\begin{array}{l}\text { ACTIVAR LOS SENTIDOS (MOTIVACION) } \\
\text { - Ambientación y saludo: Toma de asistencia } \\
\text { - Escuchar la canción "Me gusta" de Silvestre Dangond } \\
\text { - Reflexión: a partir de los sentimientos generados por la canción, se analizan las reacciones } \\
\text { de los estudiantes, respondiendo a las preguntas: } \\
\text { ¿Cuál es el mensaje que trae la canción? } \\
\text { ¿Qué sentimientos inspira? } \\
\text { ¿En qué frases de la canción se identifican figuras literarias? } \\
\text { Analizar las figuras literarias y el mensaje implícito que traen en la canción. }\end{array}$} \\
\hline \multicolumn{3}{|c|}{$\begin{array}{l}\text { LEER COMO UN ESCRIOR (LECTURA) } \\
\text { - Lectura del texto "La noche de los feos" de Mario Benedetti } \\
\text { - Identificación de saberes cotidianos: Los estudiantes responderán las siguientes situaciones } \\
\text { de forma oral: } \\
\text { iQué sentimientos inspira el texto? } \\
\text { iEn qué frases se identifican figuras literarias? } \\
\text { Analizar las figuras literarias y el mensaje implícito que traen en el texto. }\end{array}$} \\
\hline \multicolumn{3}{|c|}{$\begin{array}{l}\text { ENFOQUE COMUNICATIVO (REFLEXION LINGUISTICA) } \\
\text { - Crear el texto de manera libre de acuerdo con las necesidades de los estudiantes. } \\
\text { - Los chicos pueden escoger una frase de la canción para iniciar su propia escritura. }\end{array}$} \\
\hline \multicolumn{3}{|c|}{$\begin{array}{l}\text { LA COMPOSICION A PARTIR DE LA INTERACCION Y EL DIALOGO } \\
\text { (PLANIFICAR LA ESCRITURA) } \\
\text { - Lluvia de ideas para planificar el texto, mediante las frases de la canción que llamaron la } \\
\text { atención de los estudiantes. } \\
\text { - Identificar posibles temas del texto para iniciar la escritura }\end{array}$} \\
\hline \multicolumn{3}{|c|}{$\begin{array}{l}\text { ESCRITURA (COMPOSICION DEL TEXTO, BORRADORES) } \\
\text { - Crear un texto narrativo a partir del final del cuento leído. }\end{array}$} \\
\hline \multicolumn{3}{|c|}{$\begin{array}{l}\text { LECTURA GRUPAL Y COEVALUACION (CORRECCION) } \\
\text { - Evaluación Lectura en voz alta de los textos realizados y la respectiva corrección } \\
\text { - Compromiso: Presentar en medio magnético el texto narrativo corregido con el fín de } \\
\text { recopilar dicho material para la revista digital. }\end{array}$} \\
\hline \multicolumn{3}{|c|}{$\begin{array}{l}\text { RECURSOS } \\
\text { Canción "Me gusta" de Silvestre Dangond } \\
\text { Texto narrativo "La noche de los feos" de Mario Benedetti } \\
\text { Video beam, Sonido } \\
\text { Computador, Internet } \\
\text { Cuademo de registros }\end{array}$} \\
\hline
\end{tabular}

Fuente: Elaboración propia. 


\section{REsultados}

De acuerdo con la figura 1, solo el $13 \%$ de los estudiantes de la IE lograron ubicarse entre los puestos 1 a 200 a nivel nacional, el 21,9\% entre los puestos 201 a 400, el 26,6 \% entre los puestos 401 a 600 y el mayor promedio, el 29,2 \%, son los estudiantes ubicados en los puestos 601 a 800 .

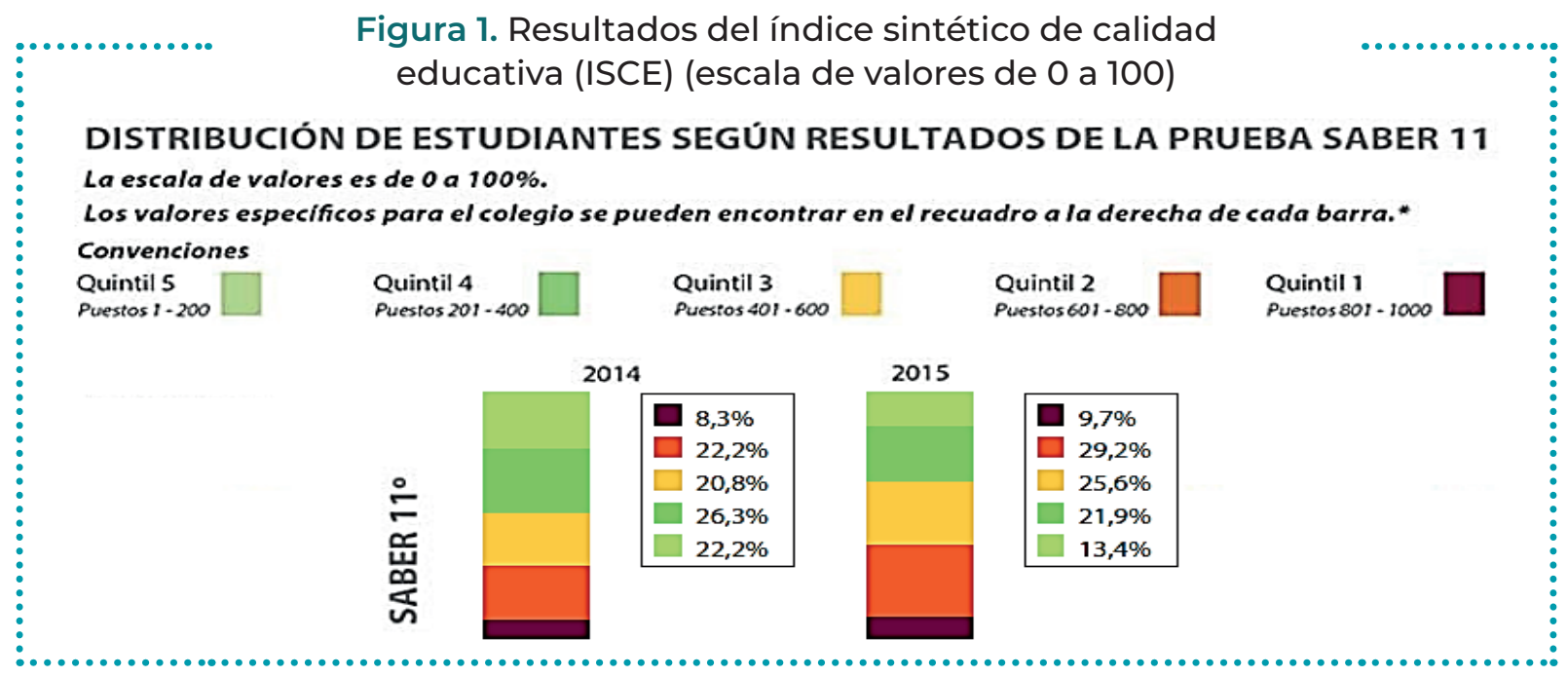

Fuente: Ministerio de Educación Nacional (2015).

En este sentido, la recolección de la información partió del problema arrojado en el análisis de los bajos resultados del ISCE en la competencia lectoescritora. Se establecieron las habilidades comunicativas con bajo desempeño en el nivel de educación media y se pensó en el diseño de actividades que las fortalezcan. De esta manera, entra en juego la indagación y observación de las clases de lengua castellana, los gustos de los estudiantes en el acto de leer y escribir, la afinidad entre estudiantes y docente, y el rol de este en el aprendizaje.

En la figura 2, se resumen los resultados obtenidos luego de la aplicación de la prueba diagnóstica, en la cual se observa que más del $50 \%$ de los estudiantes presentan un bajo desempeño para cada una de las competencias evaluadas en los niveles de lectura: literal (29\%), inferencial (39\%) y crítico (42\%). El $50 \%$ de los estudiantes muestran habilidades sintácticas y al $22 \%$ de ellos se les facilita la producción textual. 
Cuando leer y escribir conspiran con los sentidos y las palabras

se sumergen en la música el texto escrito cobra vida

Claudia Marcela Callejas Velasco, Claudia Lucia Rugeles Rey

Figura 2. Resultados de prueba diagnóstica, grado 10B

Prueba Diagnóstica 10-02
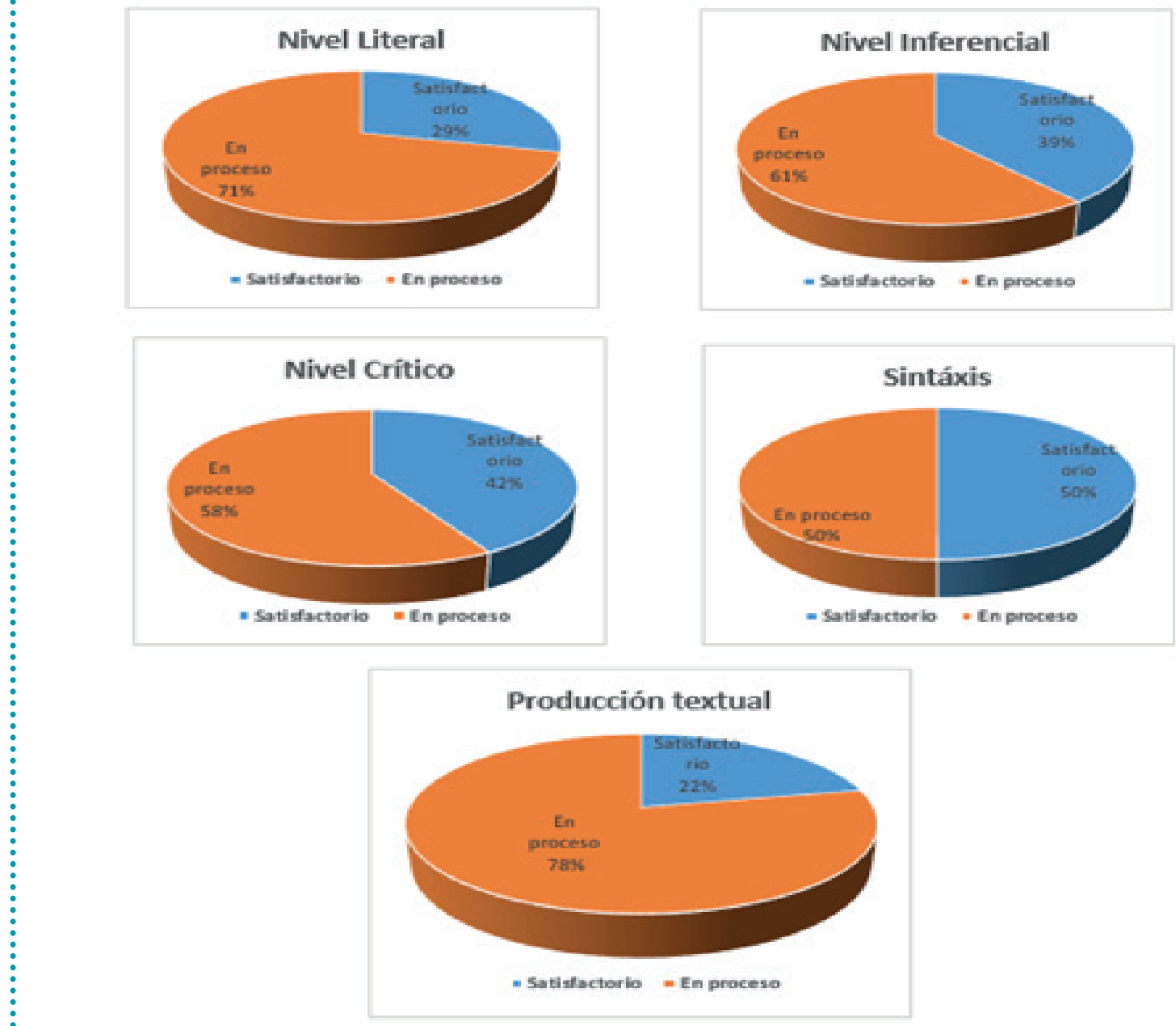

Fuente: Elaboración propia.

A partir dela la encuesta realizada en el proceso de investigación, se registraron los resultados que se ilustran en la figura 3. 


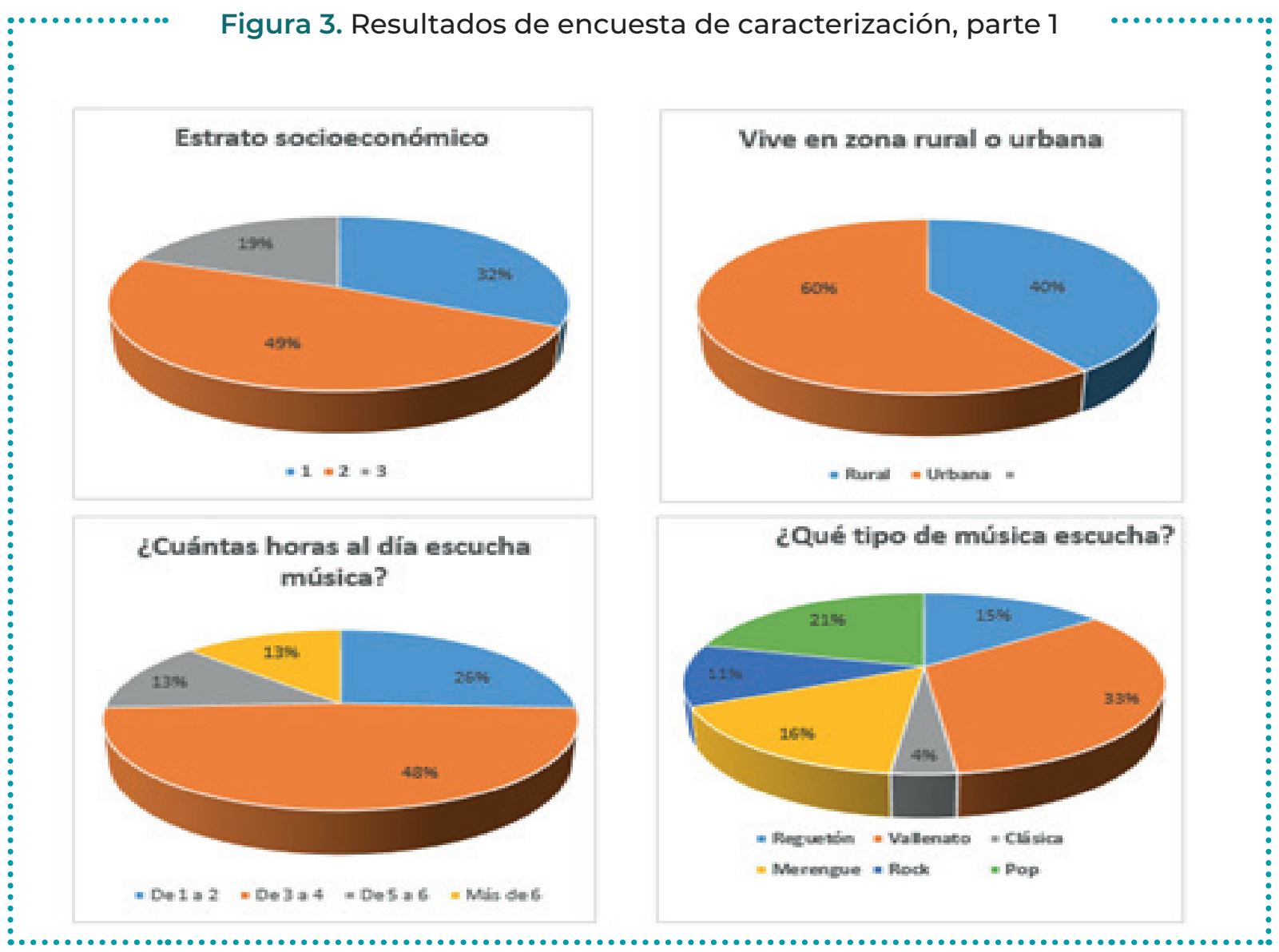

Fuente: Elaboración propia.

De acuerdo con la figura 3, que resume los resultados arrojados por la encuesta de caracterización, se observó que el $49 \%$ de los estudiantes están ubicados en el nivel 2, el $32 \%$ en el nivel 1 y el $19 \%$ en el nivel 3 de estrato socioeconómico. El $60 \%$ de los estudiantes que asisten a la IE viven zona urbana, ubicados en barrios aledaños, y el 40 \% viven en zona rural. En la anterior encuesta, se hizo necesario indagar la influencia de la música en la vida diaria, así, el $48 \%$ de los estudiantes escuchan música entre 3 y 4 horas, el $26 \%$ de 1 a 2 horas, el $13 \%$ de 5 a 6 horas y otro $13 \%$ más de 6 horas al día. Lo anterior indica que mínimamente 28 horas semanales escuchan música los estudiantes del grado 10B y evidencia variedad en sus preferencias musicales de la siguiente manera: el $33 \%$ se inclina por el vallenato, el $16 \%$ por el merengue, el $15 \%$ por el reguetón, el 
$4 \%$ por la música clásica, el $21 \%$ por el pop y el $11 \%$ por el rock. De esta manera, para el diseño de las actividades, se escogieron diferentes canciones que se relacionaran con los gustos musicales de los estudiantes (figura 4).

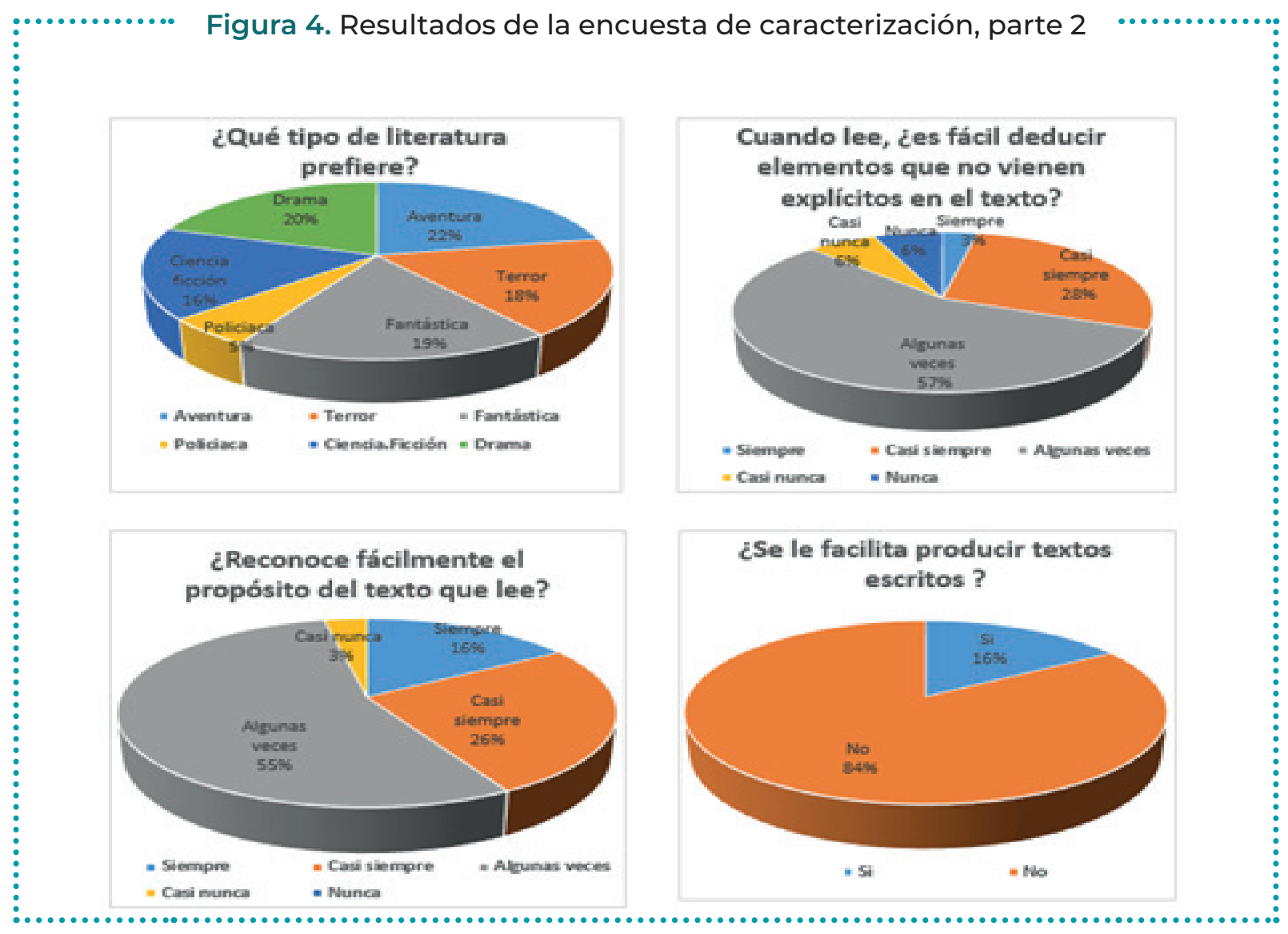

Fuente: Elaboración propia.

En la encuesta se preguntaron aspectos necesarios a considerar en la comprensión y producción textual, y se notó que, en el nivel inferencial, respecto del propósito comunicativo del texto, el $16 \%$ de los estudiantes siempre comprenden satisfactoriamente, el $55 \%$ lo logran algunas veces, el $26 \%$ casi siempre y el $3 \%$ casi nunca. Y en cuanto a la habilidad para deducir elementos implícitos en el texto, el $3 \%$ de los estudiantes respondieron que siempre lo logran, el $28 \%$ casi siempre, el $57 \%$ algunas veces, el $6 \%$ casi nunca y otro $6 \%$ nunca. 
Para atraer la atención de los estudiantes en la lectura, se indagaron sus preferencias literarias, tras lo cual se obtuvieron los siguientes resultados: al $22 \%$ les gusta leer textos de aventura, el $18 \%$ de terror, el $16 \%$ de ciencia ficción, el $20 \%$ de drama, el $19 \%$ fantástica y el $5 \%$ policiaca. Finalmente, se les preguntó a los estudiantes por la habilidad que tienen para escribir textos, a lo cual respondieron el $84 \%$ que no y un 16 \% que sí se les facilita.

En la figura 5, se registra la identificación que tuvieron los estudiantes con un tipo de escritor en particular. Después de leer y comparar las características de cada uno con su forma individual de producción para el grado 10-B, el 22 \% se identifican con Estanislao Zuleta, que es el nivel inferior de producción textual. Así, en forma ascendente, el $42 \%$ se ubica en el nivel 1, el $26 \%$ en el nivel 2 y el $10 \%$ en el nivel 3, que es el máximo nivel, en que queremos que los estudiantes lleguen al terminar la intervención pedagógica. En el grado 11-A, el $10 \%$ se identifican con Estanislao Zuleta, que es el nivel inferior de producción textual. Así, en forma ascendente, el $32 \%$ se ubica en el nivel 1, el $42 \%$ en el nivel 2 y el $16 \%$ en el nivel 3, lo que indica que los estudiantes del grado 11 tienen un poco más fortalecidas las habilidades escritoras, e implica una continuidad en el proceso para que todos lleguen al nivel superior.

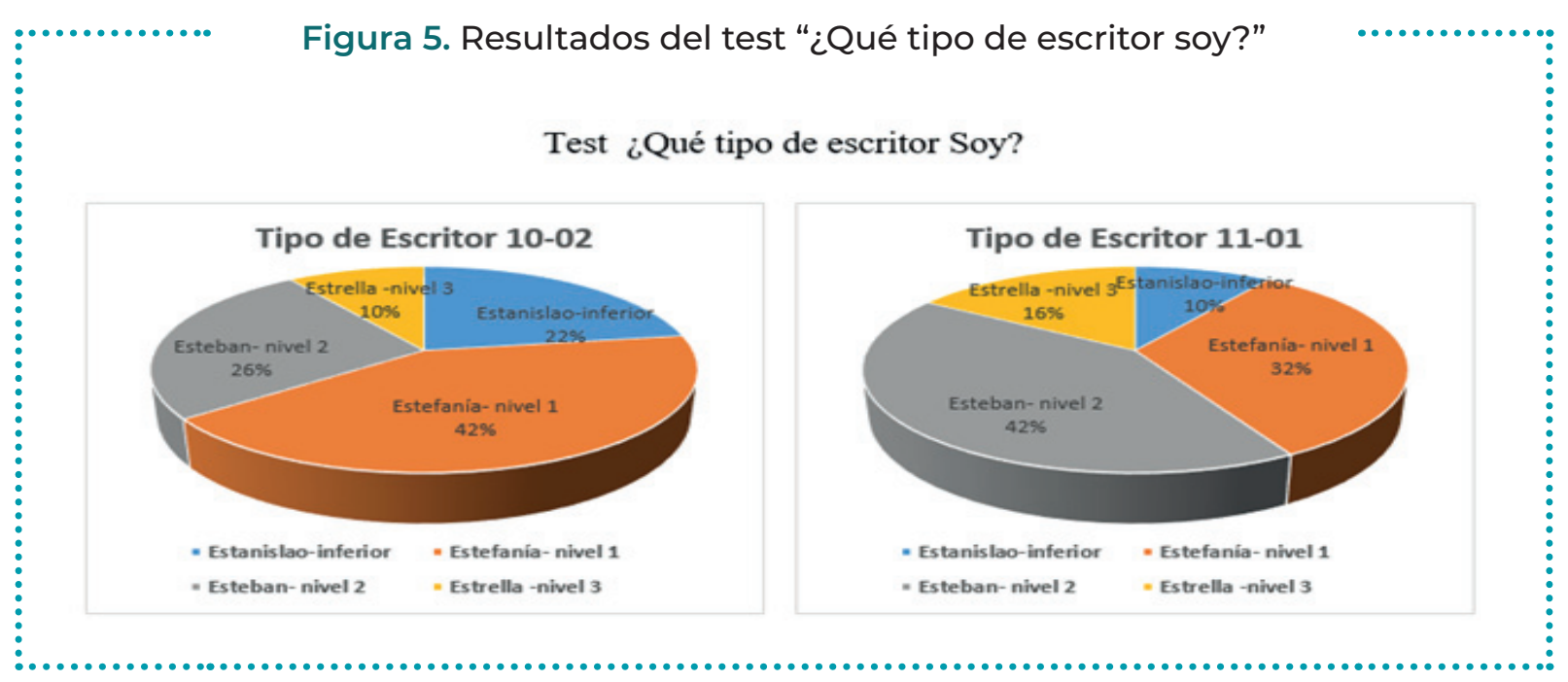

Fuente: Cassany (2014).

A partir del análisis de las categorías propuestas, relacionadas con los referentes teóricos orientados por el Ministerio de Educación Nacional (Estándares Básicos de competencias del Lenguaje, 2003) se obtuvieron grandes avances en cada una de ellas (tabla 2). 
Cuando leer y escribir conspiran con los sentidos y las palabras

se sumergen en la música el texto escrito cobra vida

Claudia Marcela Callejas Velasco, Claudia Lucia Rugeles Rey

Tabla 2. Categorías para el análisis de la investigación

\begin{tabular}{|c|c|}
\hline Categorías & Subcategorías \\
\hline La comunicación & $\begin{array}{l}\text { - Oral y escrita: } \\
\text { - Diálogo entre pares } \\
\text { - Planificación de la escritura } \\
\text { - Lectura grupal de sus producciones y coevaluación }\end{array}$ \\
\hline El sentido de la propia existencia & 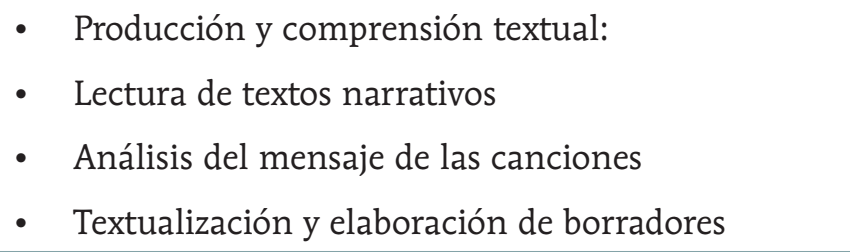 \\
\hline $\begin{array}{l}\text { La expresión de los sentimientos y } \\
\text { las potencialidades estéticas }\end{array}$ & $\begin{array}{l}\text { - Expresión de vivencias: } \\
\text { - Sentimientos } \\
\text { - Anécdotas propias } \\
\text { - Historias creadas por la imaginación }\end{array}$ \\
\hline
\end{tabular}

Fuente: MinEducación (2003).

\section{DIMENSIONES DEL LENGUAJE EVIDENCIADAS EN EL DESARROLLO DEL PROYECTO}

\section{La comunicación}

El lenguaje tiene una valía social, pues muchas de sus posibles manifestaciones, en especial la lengua, se constituyen en instrumentos por medio de los cuales los individuos dan forma y mantienen sus relaciones interpersonales, sustento y eje de la vida en comunidad. Así, formar en lenguaje para la comunicación supone formar individuos capaces de interactuar con sus congéneres, esto es, relacionarse con ellos y reconocerse (a la vez que reconocerlos) como interlocutor capaz de producir y comprender significados, de forma solidaria, en atención a las exigencias y particularidades de la situación comunicativa. Esto es, ayudar a la formación de un individuo capaz de ubicarse claramente en el contexto de interacción en el que se encuentra y estar en capacidad de identificar en este los códigos lingüísticos que se usan, las características de los participantes en el evento comunicativo, el propósito que los orienta y, según ello, interactuar (MinEducación, 2003, p. 21). 
Cuando leer y escribir conspiran con los sentidos y las palabras

se sumergen en la música el texto escrito cobra vida

Claudia Marcela Callejas Velasco, Claudia Lucia Rugeles Rey

Fuente de la investigación. Desde el enfoque comunicativo de la escritura, la comunicación es una categoría que permite su planificación. En el proceso de lectura, los estudiantes aprovechan para seleccionar las ideas más importantes y según ellas iniciar el proceso de planificación. Así lo expresa J. Pérez cuando manifiesta que "es importante sacar las ideas de los textos narrativos para empezar a escribir" (26/01/2018), lo que confirma dicha planificación. Citando otro caso, el estudiante descubre características particulares del texto que lo invita a una planeación en la que sobresalga la selección de ideas. Por ejemplo, el estudiante identifica en el cuento "Ladrón de sábado" que el autor deja la historia a la expectativa. Este hecho particular hace que él quiera escribir un texto con las mismas características. P. Rodríguez comenta: "Lo estoy empezando a planear de esa manera" (02/02/2018). Para planear la escritura, es importante aprender a leer el contexto porque este es la fuente de todas las historias. En el desarrollo de la secuencia, los estudiantes relacionaron la intención comunicativa de la letra de la canción con la del texto narrativo y sus experiencias. Al respecto, G. Martínez dice: "Ahora, cuando vemos que algo ocurre, lo imaginamos como una posible historia para escribir" (02/02/2018). En este sentido, los estudiantes notaron la necesidad de planear la escritura. Anterior a la intervención pedagógica, los estudiantes no planificaban los textos, lo cual puede ser una causa de la apatía a la escritura. Ahora, con la investigación-acción, la práctica pedagógica se renueva, el estudiante ve la necesidad de planificar el texto antes de escribirlo e integra los procesos de lectura con los procesos de planificación. El efecto que esto produce se puede resumir en la siguiente frase de I. Ramírez: "Ya no decimos que no sabemos sobre qué escribir" (09/02/2018).

Hallazgo. La escritura tiene un enfoque comunicativo, en atención a la comunicación como una categoría que permite la planificación de la escritura, en la que los estudiantes se expresan de manera oral y escrita, se evidencia que en el ejercicio de escribir es necesario brindar un buen número de horas, dedicación del docente de lenguaje, el uso de las tecnologías o herramientas didácticas, trabajo por tareas, lectura grupal, coevaluación, fomento del diálogo y la interacción.

La interacción y el diálogo permitieron conocer las apreciaciones de los estudiantes acerca de los textos leídos, el análisis del mensaje de las canciones, la relación de la historia de la canción con sus vidas, la tolerancia y el respeto hacia las situaciones de sus compañeros en las cuales basaron sus producciones. Para los estudiantes, es inquietante saber qué pasará con sus creaciones, ya que no quieren que se queden guardadas o solo como tareas de clase; por ello, el resultado final de estas composiciones es la publicación en una revista digital. 
Cuando leer y escribir conspiran con los sentidos y las palabras

se sumergen en la música el texto escrito cobra vida

Claudia Marcela Callejas Velasco, Claudia Lucia Rugeles Rey

\section{El sentido de la propia existencia}

Al poseer el lenguaje un doble valor (subjetivo y social), se constituye en una herramienta que repercute en la formación de individuos autónomos, capaces de pensar, construir, interpretar y transformar su entorno, que hace valer su condición de seres humanos únicos y diferenciados, aunque iguales a los demás en derechos, responsabilidades y potencialidades. Según las metas que aquí se han esbozado, formar en lenguaje plantea el reto de propiciar el desarrollo de las competencias que requieren los estudiantes para participar con éxito en las situaciones comunicativas que le ofrecen a diario la IE, la región, el país y el mundo, teniendo presente que ser competente en lenguaje supone el manejo de unos saberes referidos al sistema lingüístico, la producción y la comprensión textual, los contextos y la intencionalidad de los interlocutores, los universos emocionales de quienes se comunican, la cultura propia y las construidas por otros seres humanos, y los recursos ideológicos de que hace uso toda elaboración de discurso, como la adopción de una postura ética frente al discurso, los contextos y los individuos (MinEducación, 2003).

Fuente de la investigación. En la categoría del sentido de la propia existencia, el MinEducación establece que el lenguaje posee un doble valor (subjetivo y social). En este sentido, S. Torres argumenta: "Leyendo el texto de las grietas, me doy cuenta de que hay que sacar provecho de cada situación" (02/03/2018), lo que muestra claramente la relación entre el texto leído y las experiencias de vida. De igual forma, se sustenta esta relación con la apreciación que D. Jiménez manifiesta: "El ladrón no robaba por necesidad como lo hacen muchos en nuestro contexto" (02/03/2018).

El MinEducación (2003) refiere "la formación de individuos autónomos", en este sentido C. Rojas afirma: "Admiro la gente que razona y ve más allá" (16/03/2018); aquí la fuente es consciente de lo que implica pensar diferente y transformar la realidad. En esta dimensión del lenguaje, el MinEducación establece "los universos emocionales de quienes se comunican como un saber referido al sistema lingüístico", así, E. Contreras lo relaciona con "empleamos la música para animarnos" (23/03/2018). Otro saber referido es el de "los contextos" en los ejercicios de escritura, en relación con lo cual D. Arias manifiesta: "Compartimos la misma historia y los mismos sentimientos" (06/04/2018), es decir, se observa claramente la relación entre los textos que leyeron los estudiantes, los mensajes de las canciones y sus vivencias.

Hallazgo. Desde la perspectiva de la lectura del texto narrativo, escuchar música se asocia a los sentimientos o a las emociones que se generan en el oyente. El texto musical despertó diversos sentimientos; los mensajes de las canciones dispararon la capacidad de producir escritura asociada con las historias de su vida. La relación que existe con las historias que cuentan los textos narrativos y las canciones permitió que los estudiantes elaboraran juicios sobre los mensajes, temas 
Cuando leer y escribir conspiran con los sentidos y las palabras

se sumergen en la música el texto escrito cobra vida

Claudia Marcela Callejas Velasco, Claudia Lucia Rugeles Rey

y hechos de la vida real; de esta manera, se les facilitó extraer ideas para abordar en sus propias composiciones, sintieron satisfacción de sus propios escritos, incluso, los compararon en su forma con la de nuestro nobel de literatura.

Los escritos de los estudiantes pasaron por un proceso de textualización, que inició en la planificación, luego la elaboración de borradores y finalmente la corrección; este paso a paso para la composición escrita facilitó el ejercicio de producción textual. Inicialmente, se pensó en abordar la producción de diferentes textos, pero durante el proceso notamos que con esta variedad no conseguíamos evaluar directamente el avance y quedaría muy amplia para abarcar en las actividades; por ello, se pensó solo en el texto narrativo por la proximidad que tiene con las historias de vida de los estudiantes.

\section{La expresión de los sentimientos y las potencialidades estéticas}

El lenguaje permite la creación de una representación conceptual de la realidad y, a la vez, ofrece la oportunidad de darle forma concreta a dicha representación, o bien de manera tendiente a la "objetividad", como en el discurso técnico y científico, o bien de manera "subjetiva", con lo cual surgen, entre otras, las expresiones emotivas y artísticas. Así, diversas manifestaciones del lenguaje le brindan al individuo la posibilidad de expresar sus sentimientos más personales, en modalidades como el diario íntimo, la literatura, la pintura, la música, la caricatura, el cine, la escultura. Formar en el lenguaje para la expresión artística implica, pues, trabajar en el desarrollo de las potencialidades estéticas del estudiante, esto es, propiciar el reconocimiento de las posibilidades significativas que le ofrece el lenguaje, por medio de sus distintas manifestaciones, para formalizar su manera particular de ver y comprender el mundo, y así recrearlo y ofrecerlo a otras miradas y perspectivas (MinEducación, 2003).

Fuente de la investigación. En atención a la dimensión de expresión de sentimientos y potencialidades que refiere el MinEducación (2003), se encuentra que "el lenguaje permite la creación de una representación conceptual de la realidad y, a la vez, ofrece la oportunidad de darle forma concreta a dicha representación" (p. 22). Durante las actividades de la intervención, se notó que este planteamiento se evidencia en la realidad próxima de los estudiantes cuando realizaron análisis de textos y los compararon con las vivencias propias o ajenas, como lo argumenta C. Zambrano: "Desde el punto de vista psicológico, siento que en el texto narrativo el ladrón tiene falta de afecto, pues se nota que roba solo el fin de semana; como no tendrá una familia con la que pasar buenos momentos, decide ir en busca de aventuras".

La representación de la realidad en este caso mediante la escritura puede tender a la "objetividad", por ejemplo, en el discurso técnico y científico, o de manera "subjetiva", con lo cual surgen, entre 
Cuando leer y escribir conspiran con los sentidos y las palabras

se sumergen en la música el texto escrito cobra vida

Claudia Marcela Callejas Velasco, Claudia Lucia Rugeles Rey

otras, las expresiones emotivas y artísticas, como puede leerse en C. Zambrano: "Le pido a Dios que algún día perdone a mi madre, pues de pequeña me hizo hacer muchas cosas, por medio de estas actividades, de las historias y apoyo de mis compañeros y de todo lo que escribí, me desahogué y tomé mucho valor, quiero salir adelante por mis hermanos, quiero cambiarles la vida y mi pensamiento será siempre: iSigue adelante y deja atrás tus tormentos!".

Hallazgo. El aprendizaje no es un hecho separado de los sentimientos. En este sentido, las composiciones escritas nacieron de los intereses de los estudiantes y generalmente desde las propias experiencias de vida que tocan los sentimientos y las emociones, a veces difíciles de expresar. Una anécdota, una situación triste o difícil, un anhelo o una gran aventura, fueron plasmadas como una historia con estructura narrativa real o imaginaria. La inteligencia emocional desempeñó un papel importante en la productividad escolar, permitió el desarrollo de habilidades necesarias en el acto de escribir y conectó los sentimientos y el contexto.

\section{CONCLUSIONES}

La investigación participativa facilitó conocer detalladamente un problema que aqueja a la mayoría de IE del país, y que en este caso no es la excepción; gracias a las características de este tipo de investigación, que permitieron realizar la intervención pedagógica durante el proceso, pudo lograrse el objetivo inicial.

La inteligencia emocional ocupa gran parte en la vida del ser humano, desde una pequeña tarea hasta la toma de decisiones; se es más emocional que racional, cuando una persona maneja sus emociones, es más eficaz para hacer sus deberes. En este sentido, Goleman (2007) resalta que el aprendizaje no es un hecho separado de los sentimientos: "ser un alfabeto emocional es tan importante para el aprendizaje como la instrucción en matemática y lectura” (pág.331).

Basándonos en ello, el desarrollo de las secuencias didácticas inició con la motivación generada por las canciones que activaron los sentimientos y los recuerdos de los estudiantes, y despertó el deseo de plasmar aquellas historias de vida convertidas finalmente en textos narrativos.

La propuesta planteada tuvo acogida por los estudiantes, ya que promovió espacios de socialización, respeto, cooperación y participación armónica. De esta manera, se mejoraron situaciones que inhibían la adquisición de estrategias de composición oral y escrita, y se evidenciaron potencialidades comunicativas textuales desconocidas; tarea difícil para los docentes en el área de lengua castellana. 
Cuando leer y escribir conspiran con los sentidos y las palabras

se sumergen en la música el texto escrito cobra vida

Claudia Marcela Callejas Velasco, Claudia Lucia Rugeles Rey

En el momento de la lectura grupal y coevaluación, para los estudiantes es inquietante saber qué pasará después con sus textos; por ello, se ideó su publicación en una revista digital, lo que indica que la propuesta para fortalecer la producción textual generó excelentes resultados.

La oralidad constituye la base comunicativa previa desde la que el aprendiz desarrolla lo escrito. El diálogo es el instrumento más eficaz para regular los procesos de análisis, reflexión y valoración que requiere. Cuando el alumno habla de lo que está escribiendo, verbaliza su pensamiento interno y permite que otras personas, compañeros y docentes le ayuden a conformarlo, que le hagan preguntas que le animen a ampliarlo, o que le muestren formas alternativas de pensamiento que no conocía (Cassany, 2014, p. 143).

En la planificación de las actividades, inicialmente se pensó fortalecer solo la producción textual, pero, en atención a los aportes teóricos consultados, se notó que en sus planteamientos la lectura y la escritura son dos procesos que van de la mano, así como referencia Cassany (2006, pág. 138): "leer y escribir son una pareja de baile". Por ello, al escoger las canciones, los textos que las apoyaban debían tener relación con el mensaje de la canción, porque antes de escribir es necesario leer como un escritor.

Para la composición escrita, es fundamental dedicar un buen número de horas de clase, ya que es un proceso riguroso que requiere dedicación del maestro y una planeación especial basada en las actividades que desarrollen las habilidades escritoras y no en ejes temáticos.

La mayoría de los adolescentes se sienten muy inseguros cuando tienen que explicar algo e incluso aceptan su incapacidad. Esto no es bueno. Hay que darse cuenta de que redactar correctamente - lo cual no es un indicio de sensibilidad literaria- es ante todo un problema técnico y que debe resolverse a tiempo para que no se convierta en un problema psicológico. (Cassany, 1995, p. 13)

Las historias de vida de los estudiantes son un tesoro para ellos, por tanto, se requiere el compromiso ético y moral del docente de salvaguardar su identidad y consentimiento de sus representantes legales para ser divulgadas, aunque se trate de textos que en algunos casos pueden ser producto de la imaginación.

Finalmente, de acuerdo con Cassany (2017), el código escrito se aprende básicamente a través de la lectura por placer. Demuestra mediante algunos estudios que hay una relación directa entre el hábito y el placer por la lectura, y la competencia en la expresión escrita. "El hábito y el placer de la lectura incrementan tanto la comprensión como la expresión escrita" (p. 61). 


\section{REFERENCIAS}

Bohórquez, J. C. (2016). La canción como estrategia didáctica para desarrollar la comprensión lectora (Tesis de grado, Universidad Distrital Francisco José de Caldas, Bogotá, Colombia). Recuperado de http://repository.udistrital.edu.co/bitstream/11349/3402/1/BohorquezJuanCarlos2016.pdf

Botello Carvajal, S. M. (2013). La escritura como proceso y objeto de enseñanza (Tesis de maestría, Universidad del Tolima, Ibagué, Colombia). Recuperado de http://repository.ut.edu.co/handle/001/1039

Cassany, D. (1989). Describir el escribir. Barcelona, España: Paidós.

Cassany, D. (1995). La cocina de la escritura. Barcelona, España: Anagrama.

Cassany, D. (2014). Construir la escritura. Barcelona, España: Paidós.

Cassany, D. (2017). Describir el escribir: cómo se aprende a escribir. Bogotá, Colombia: Paidós.

Bachmann, M. L. (1988). La rítmica Jaques Dalcroze: una educación por la música para la música. Madrid, España: Pirámide.

Díaz, M. L., Morales Bopp, R. y Díaz Gamba, W. (2014). La música como recurso pedagógico en la edad preescolar. Infancias Imágenes, 13(1), 102-108. https://doi.org/10.14483/16579089.5455

Goleman, D. (2007). La inteligencia emocional. Ciudad de México, México: Ediciones B.

Ministerio de Educación Nacional. (2003). Estándares básicos de competencias del lenguaje: formar en lenguaje. Apertura de caminos para la interlocución. Bogotá, Colombia: Autor. 